DIALYSIS

\title{
Dialyzer sterilization method linked to thrombocytopenia
}

Use of dialyzers sterilized by electronbeam radiation is associated with an increased risk of thrombocytopenia, according to new findings. Mercedeh Kiaii and colleagues initiated their study to investigate the association between thrombocytopenia and use of electronbeam-sterilized dialyzers after identifying 20 index patients in their dialysis unit who had thrombocytopenia following exposure to electron-beam-sterilized dialyzers. "What initiated the study was the identification of cases with unexplained significant thrombocytopenia that were clearly associated with the dialysis treatment and would resolve when the dialyzer was changed to one that was not sterilized with electron-beam sterilization," explains Kiaii.

To further investigate this association, the researchers used provincial data on patients undergoing hemodialysis in British Columbia and Alberta to assess the prevalence and possible cause of thrombocytopenia in these two provinces. Using historical data, the researchers found that the prevalence of thrombocytopenia in British Columbia had increased after September 2009, coinciding with a province-wide switch to the predominant use of dialyzers sterilized by electron-beam radiation.

\section{4 ...thrombocytopenia is} difficult to diagnose ... because ... platelet counts are not routinely measured... 77

The researchers then prospectively analyzed predialysis and postdialysis platelet counts in both populations. They found that thrombocytopenia was more prevalent in patients using electron-beamsterilized dialyzers than in patients using non-electron-beam-sterilized dialyzers. A logistic regression analysis revealed that use of an electron-beam-sterilized dialyzer was associated with an increased risk of thrombocytopenia.

These findings led both provinces to stop using electron-beam-sterilized dialyzers. Over the ensuing 6 months, the researchers noted a $50 \%$ decrease in the percentage of patients with postdialysis thrombocytopenia. In British Columbia, the percentage of patients who had thrombocytopenia, defined as a postdialysis platelet count of $<100 \times 10^{3} /$ $\mu \mathrm{l}$ and $\mathrm{a}>15 \%$ decrease in platelet count from predialysis levels, declined from 7.2\% (95\% CI 6.0-8.6\%) to $2.1 \%$ (95\% CI 1.5-2.9\%) after use of electron-beamsterilized dialyzers was stopped. A similar pattern was observed in Alberta.

The researchers say that thrombocytopenia is difficult to diagnose in hemodialysis patients because postdialysis platelet counts are not routinely measured in clinical practice. They also note that their study does not identify a mechanism for the association between sterilization method and thrombocytopenia. "As patient safety was the first priority, we completely discontinued the use of electron-beam dialyzers but believe that further studies need to be conducted to evaluate what possible changes occur in dialyzer membrane integrity that may lead to platelet activation, aggregation and thrombocytopenia," says Kiaii.

Susan J. Allison
Original article Kiaii, M. et al. Use of electron-beam sterilized hemodialysis membranes and risk of thrombocytopenia. JAMA 306, 1679-1687 (2011) 